\title{
Review of treatment outcome of multi-drug resistant tuberculosis
}

\author{
Prashanth Chikkahonnaiah", ${ }^{1, *}$ H.M. Virupaksha ${ }^{2}$ \\ ${ }^{\mathbf{1}}$ Assistant Professor, ${ }^{2}$ Professor, Mysore Medical College and Research Institute, Mysuru, Karnataka, India
}

*Corresponding Author:

Email: prshnthcr@gmail.com

\begin{abstract}
Introduction: Multidrug resistant tuberculosis (MDR TB) is essentially a man-made phenomenon and arises mainly due to inadequate treatment of drug-sensitive TB. Drug resistance is seen in 1-3\% of new cases and $12 \%$ of re-treated tuberculosis.

Material and Methods: We studied 291 cases of multidrug resistant tuberculosis registered at drug resistant tuberculosis (DRTB) center, Mysore. Patients were treated according to programmatic management of drug resistant tuberculosis (PMDT) guidelines by DRTB committee of the hospital.

Results: Out of 291 treated cases of MDR TB, 41 were diagnosed when they were on Category I regimen of revised national tuberculosis control program. Thirty cases were treatment after lost to follow-up. Twenty-one cases were co-infected with tuberculosis and human immune deficiency virus.

Conclusion: Treatment outcome was available in 275 patients. Out of which 16 cases completed treatment, 106 were cured, 55 cases stopped treatment, 84 died, treatment was stopped in 3 patients due to some reason and 11 cases were switched to extensively drug resistant tuberculosis (XDR) regimen. Our study concluded that despite adequate drugs with well-organized program the treatment outcome of MDR tuberculosis was still low.
\end{abstract}

Keywords: Multidrug tuberculosis; Drug resistant tuberculosis center; Programmatic management of drug resistant tuberculosis; Extensively drug resistant tuberculosis.

\section{Introduction:}

India has more than $25 \%$ of world's tuberculosis incidence cases. Every day more than 1000 Indians die of TB [1]. Multidrug resistant tuberculosis (MDR-TB) is defined as a suspect who is sputum culture positive and has Mycobacterium tuberculosis resistant to isoniazid and rifampicin, with or without resistance to other anti-tubercular drugs based on results from an RNTCP accredited laboratory. A patient who is resistant only to rifampicin is also treated with same regimen as MDR tuberculosis. 1-3\% of new cases and $12 \%$ of retreatment cases are estimated to have drug resistant tuberculosis [2]. Despite well-organized programmatic management of drug resistant tuberculosis (PMDT) program the cure rates of MDR tuberculosis is often low. The present study tries to understand various treatment outcomes of MDR tuberculosis.

\section{Material and Methods:}

The study was conducted by the Department of Pulmonary Medicine in the Princess Krishnajammanni Tuberculosis and Chest Diseases (PK TB \& CD) hospital, and Mysore Medical College and Research institute.

It was a retrospective study done on patients who were admitted in DRTB center of PK TB \& CD hospital, Mysore for initiation of treatment. All the cases of MDR tuberculosis who were admitted in PK TB \& CD hospital for initiation of treatment from 2012 December to January 2015 were included in the study. MDR tuberculosis patients who were started treatment in some other DRTB center and admitted in PKTB hospital for management of complication or adverse effects were excluded from the study.

Standard protocol to be followed: Available data from in-patient records of all MDR TB cases were collected. For patients getting treated under PK TB \& CD DRTB center, treatment outcome shall be verified by culture conversion reports. For patients getting treated under another DRTB center, details about the treatment status and outcome of the disease shall be enquired from respective tubercular unit staff.

\section{Results:}

Total 291 patients were enrolled for the study. Out of 291 patients 222 were male and 69 were female. The mean age of the patients was 40.9 years. 21 patients were TB-HIV coinfection cases. 47 patients had diabetes. 2 patients had preexisting liver disease. 2 patients had preexisting psychiatric illness 1 patient had chronic kidney disease. Other 218 patient had no other significant medical illness.

Antitubercular treatment history: 39 patients were diagnosed as MDR tuberculosis while they were on category I of anti-tubercular treatment. The most common group of patients with MDR tuberculosis was treatment failure (161) and treatment after default (30). Thus MDR tuberculosis is more common in retreatment cases than in primary cases.

Weight band: 5 patients were of the weight band 1625,165 patients were of the weight band 26-45, 106 patients were of the weight band 46-70 and 4 patients were more than 70 kilogram. Tuberculosis being a 
wasting disease, most of the patients were below the average body weight of healthy individuals of the country.

Modified MDR regimen: For the patient, who had chronic kidney disease, anti-tubercular drugs were started as per creatinine clearance and Kanamycin was replaced with Para-amino salicylic acid (PAS). One patient had hearing loss before initiation of treatment for which Kanamycin was replaced with PAS. One patient with jaundice was treated without any drug modification after liver function test normalized. For one patient with cirrhosis was treated by eliminating Pyrazinamide and Ethionamide. Two patients with preexisting psychiatric illness were given MDR drugs along with antipsychotics.

Patients requiring readmission: 59 patients were readmitted to the DRTB center with few patients requiring more than one admission. Out of which 16 were admitted for breathlessness, 12 for hearing problem, 6 for gastritis, 6 for hemoptysis, 6 for irregular treatment, 4 for psychosis, 4 for deranged RFT, 3 for initiation of XDR regimen, 2 for pneumothorax, 2 for joint pain, 2 for skin reaction, 1 for GTCS and 1 for myeloneuropathy. All patients were managed successfully for the adverse drug reactions. And patients on irregular treatment were reinforced the importance of adherence to treatment.

Drug regimen changes: Kanamycin was the most offending drug with 17 patients developing significant side effects leading to replacement of Kanamycin. 12 patients had hearing loss, 3 patients refused Kanamycin, 1 patient developed injection site abscess and 1 patient developed renal failure. Three patients developed severe psychiatric symptoms requiring change of Cycloserine. Pyrazinamide was stopped in 1 patient due to severe joint pain. Ethambutol was stopped in one patient with visual disturbance. Ethionamide was stopped in one patient due to psychiatric symptoms.

Treatment outcome: 106 patients were cured and 16 completed treatment. 84 patients died during the course of the treatment. 55 patients stopped treatment. Out of these 55 patients, 10 were admitted to hospital earlier with some adverse reaction. 11 patients were diagnosed as extensively drug resistant tuberculosis and were switched to XDR regimen. Treatment was stopped in 3 patients due to some reason. And treatment outcome of 16 patients were not available as they were either still on treatment or no data for verification.

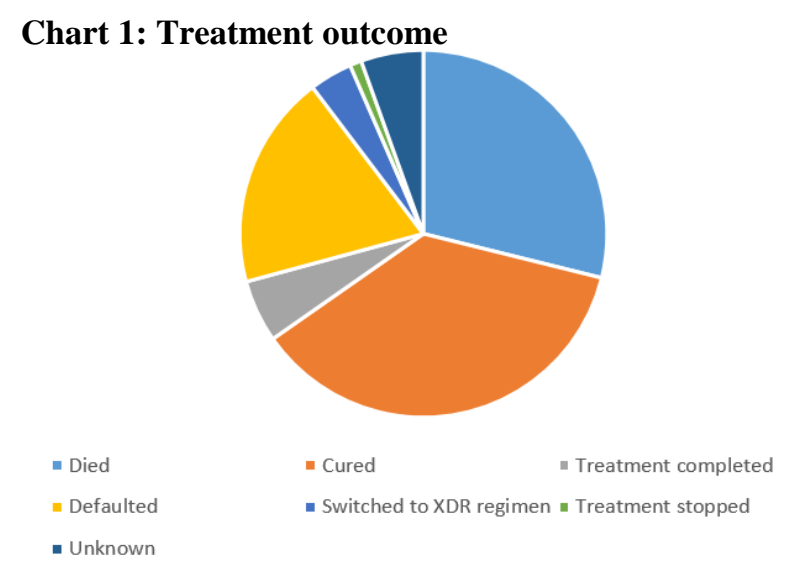

HIV and MDR: 21 patients were co-infected with HIV and tuberculosis out of which 3 were female and 18 were male. 11 patients died during the course of the treatment while 3 patients were lost to follow up and 5 patients were cured.

Death: 84 patients died during the course of the treatment, exact cause of death was not ascertained. There were 9 institutional deaths of which 7 were due to respiratory failure, 1 was due to hemoptysis and 1 was due to pneumothorax.

\section{Discussion:}

Tuberculosis has become a global emergency and World Health Organization (WHO) has declared tuberculosis as a notifiable disease [3]. In India incidence of tuberculosis was estimated to be 171 per lakh population, prevalence was 211 per lakh population with mortality varying between $12-28$ deaths per lakh population in various regions [4]. RNTCP made a significant progress and achieved its goal of $70 \%$ case detection rate and $85 \%$ cure rate [5]. It is to be realized that even after achieving $70 \%$ case detection and $85 \%$ cure rate, we are actually curing $59 \%$ patients. So, another $41 \%$ of the patients in the community remain sputum positive and spread the disease. And outcomes other than cure are alarming for the community. Raising cases of death, defaulter and failure should rouse suspicion of emergence of MDR tuberculosis. The prevalence of MDR-TB mirrors the functional state and efficacy of tuberculosis control programs in the country. To meet the challenge of MDR tuberculosis, DOTS plus (now called as programmatic management of drug resistant tuberculosis) was introduced.

Present study tried to throw light on various aspects of MDR tuberculosis. MDR tuberculosis was more common in males. This probably is because most of the rural females are house wife and do not come in contact with many people. MDR tuberculosis was more common in retreatment cases rather than new case. Thus well managed tuberculosis control program is the need of time. Most of the patients were of the weight band 26-45. This could be due to two reasons. Firstly 
tuberculosis is common in malnourished people. And also tuberculosis being a chronic illness leads to cachexia.

Less than 10 patients required initial modification in treatment regimen. For patients with preexisting kidney disease Inj. Kanamycin was replaced with PAS. For patients with preexisting liver disease Pyrazinamide and Ethionamide were avoided. For patients with preexisting psychiatric illness Cycloserine was avoided. Most of the patients tolerated drugs well. Common side effects encountered were hearing problem, gastritis, hemoptysis, psychosis, deranged renal function tests, joint pain, skin reaction while generalized tonic clonic seizures and myeloneuropathy were rare side effects.

Dhingra et al., conducted a study of 27 proven cases of MDR tuberculosis. Of the 27 patients, 13 were cured, 10 defaulted, one died, one was still on treatment and two were referred for surgery. This study shows the poor treatment outcome of MDR tuberculosis [6].

In another study conducted by Joseph et al., 38 MDR patients were enrolled for the study. At the end of treatment, 25 (66\% were cured, $5(13 \%)$ defaulted, 3 $(8 \%)$ had died and $5(13 \%)$ had failed treatment. Of the three deaths two died out of reasons other than tuberculosis. Out of 5 who had defaulted, two showed XDR pattern [7].

Anderson et al., conducted a retrospectiveprospective cohort study on 204 cases of MDR tuberculosis. Of the 204 cases studied only 56 (30.4\%) patients had previous history of tuberculosis. Totally $144(70.6 \%)$ patients successfully completed treatment. 13 patients died out of tuberculosis, 16 were lost to follow up, 1 patient relapsed, 10 patients had neutral reports and 9 were transferred overseas [8].

Our study enrolled 291 MDR tuberculosis cases and 275 patients had some outcome. Among 220 patients who were on regular treatment 122 (55.4\%) were either cured or completed treatment. However the cure rates of HIV-MDR tuberculosis was quite low $(23 \%)$ and death rates were high $(52 \%)$. Considering the long duration of the treatment, adherence to treatment was a major issue and 55 patients $(18.9 \%)$ stopped treatment. The default rates could probably be reduced by short course drug regimen and proper management of adverse reactions at treatment provider site. 11 patients were switched to XDR regimen. However whether patients were having pre-existent XDR tuberculosis could not be ascertained. Thus patients with drug resistant tuberculosis must be screened for resistance to second line drugs before starting treatment to avoid further progression of the disease.

Thus MDR tuberculosis is a manmade phenomenon. Properly managed RNTCP program may prevent emergence of MDR tuberculosis. Patients tolerate MDR treatment well, provided timely management of adverse reactions were done. Adherence to treatment is the key for success even for
PMDT program. However treatment outcome of MDR tuberculosis is often poor. And treatment outcome in HIV positive patients were much lower. Initial evaluation for resistance of both first and second line drugs may improve outcome of treatment. With present availability of rapid diagnostic test to detect resistance to second line drugs and shorter MDR regimen, treatment outcome may improve in future.

Conflicts of interests: None declared

Acknowledgements: None

\section{References:}

1. Behera D. Textbook of Pulmonary Medicine. Jaypee Brothers Publishers; 2010. 1651 p.

2. Chhabra N, Aseri ML, Dixit R, Gaur S. Pharmacotherapy for multidrug resistant tuberculosis. J Pharmacol Pharmacother 2012;3(2):98-104.

3. Bhaumik $\mathrm{S}$, Biswas T. India makes tuberculosis a notifiable disease. CMAJ Can Med Assoc J 2012;184(10):E519-20.

4. WHO | Global tuberculosis report 2014 [Internet]. WHO. [cited 2015 Oct 2]. Available from: http://www.who.int/tb/publications/global_report/en/

5. Bhattacharya S, Singh A. Why tuberculosis control programmes fail? Role of microlevel and macrolevel factors: an analysis from India. BMJ Case Rep 2017;2017:bcr-2017-219606.

6. Dhingra VK, Rajpal S, Mittal A, Hanif M. Outcome of multi-drug resistant tuberculosis cases treated by individualized regimens at a tertiary level clinic. Ind $J$ Tuberc 2008;55(1):15-21.

7. Joseph P, Desai VBR, Mohan NS, Fredrick JS, Ramachandran R, Raman B, et al. Outcome of standardized treatment for patients with MDR-TB from Tamil Nadu, India. Ind J Med Res 2011 May;133:529-34.

8. Anderson LF, Tamne S, Watson JP, Cohen T, Mitnick C, Brown $\mathrm{T}$, et al. Treatment outcome of multi-drug resistant tuberculosis in the United Kingdom: retrospectiveprospective cohort study from 2004 to 2007. Euro Surveill Bull Eur Sur Mal Transm Eur Commun Dis Bull 2013;18(40):8.

How to cite this article: Chikkahonnaiah $P$, Virupaksha H.M. Review of treatment outcome of multi-drug resistant tuberculosis. IP Indian $J$ Immunol Respiratory Med 2018;3(3):114-116. 